\title{
Studies on the optical fluxes attenuation process in optoelectronic systems
}

\author{
T.A. Strelkova \\ Kharkiv National University of Radio Electronics, 61166 Kharkiv, Ukraine \\ E-mail: strelkova_t@ukr.net
}

\begin{abstract}
In this paper, the statistical characteristics of output signals in optoelectronic systems with a limited dynamic range are considered. The experimental results concerning noises probability densities are adduced. It has been shown that the law of output signal distribution depends on attenuation factor of neutral filter used for matching the dynamic range. The output signal distribution law is well approximated by the steady distributions with characterization index $0<\alpha<2$. Using the stable law as a limiting one allows to avoid the contradiction between experimental data and common mathematical models of signals based on the Poisson and Gaussian statistics and to reduce weak signals detection errors in optoelectronic systems with implementation of neutral filters for matching the dynamic range.
\end{abstract}

Keywords: optical signal detection, optical attenuator, optical filter, photodetector, noise measurement, stochastic process, statistical analysis, analysis of variance, statistical distribution.

Manuscript received 14.05.14; revised version received 19.08.14; accepted for publication 29.10.14; published online 10.11.14.

\section{Introduction}

In recent years, the optical and optoelectronic systems occupy leading positions in astronomical, medical and biological researches. For example, carrying out the astronomical researches in night and day time conditions, discovery of new space objects, obtaining more detail information about physiological features of tumor and bone tissues, hemolysis of erythrocytes, productive capacity of sperm cells [1-3]. The analysis of the obtained images includes methods of filtering and improvement of image quality with the aim to detect useful objects. The optoelectronic systems operate under different conditions of illumination providing the system operation in linear and non-linear modes at the boundaries of the dynamic range. Neutral and spectral filters are widely applied when working at the upper limit of the dynamic range for coordination of the dynamic range of optical receivers with a level of the received optical signal, alongside with usage of different circuits and methods for automatic regulation of the input optical radiation attenuation. However, when using attenuators in the composition of optoelectronic systems, the probabilistic nature of weakening the input optical radiation causes additional fluctuations, which results in the relative increase in its dispersion as compared to the mean value. The increase in the attenuated flux dispersion renders an essential effect on the characteristics of useful signal detection.

The report on the performed experimental researches aimed at the process of the optical flux weakening in optoelectronic systems is presented in this paper. The researches include studying the statistical characteristics of the optoelectronic system output signals, when using different levels of weakening the input optical fluxes. The effect of output signal statistics on the main specifications of image quality has been analyzed. 


\section{Experimental}

The studies were conducted in laboratory and full-scale conditions. The schemes of our installations are shown in Fig. 1. The optical signal of low intensity was applied to the input of the system, a photo electronic multiplier (PEM) operated in the photon counting mode. To decrease the internal noise effect, PEM was preliminary kept in the dark. The probabilistic beam attenuation was carried out using neutral filters with the specified optical density (Fig. 1a).

The values $\psi_{\text {in }}$ and $\psi_{\text {out }}$ were measured:

$\psi_{\text {in }}=\frac{u_{\text {in }}}{\sigma_{\text {in }}}, \psi_{\text {out }}=\frac{u_{\text {out }}}{\sigma_{\text {out }}}$,

where $u_{\text {in }}$ and $u_{\text {out }}$ are the mean values of the input and output fluxes, $\sigma_{i n}$ and $\sigma_{\text {out }}$ - root-mean-square deviations of the input and output fluxes.

The results of the experimental measurements of values $\psi_{\text {in }}$ and $\psi_{\text {out }}$ are obtained when averaging over 10000 realizations. Fig. 2 demonstrates the results of the normalized value $\psi=\psi_{\text {out }} / \psi_{\text {in }}$.

Radiation receiver properties, propagation medium properties, optical link properties and optical radiation corpuscular properties render effect on statistical parameters of signals. Analyzing the statistical characteristics of the light field, one should take certain physical and mathematical, statistical models. Making mathematical models of the received optical radiation, one should use normal, log-normal, exponential, distribution of Bose-Einstein. The mathematical models of the received signals based on Poisson statistics were widely spread [4-8]. These models take into account the corpuscular structure of the photon flux, conditions of formation of optical radiation, the lack of interaction between photons in the flux, and also the stochastic properties of optical signals when analyzing quantum noise. The Poisson flow model was used for calculation of theoretical dependence of the normalized value $\psi$ (Fig. 2).

The analysis of Fig. 2 shows that the results of experimental researches demonstrate deviations from adopted theoretical Poisson model with increasing the attenuation factor. It is possible to explain these changes by the increase in the root-mean-square deviation of the registered flux as compared to its average value. It is shown in [9-11], when studying the statistics of photons, that the fluctuations of the received optical radiation also differ from the adopted Poisson and Gaussian models. The research demonstrates a change in the received signal dispersion relative to the mean value and the use of the sub-Poisson statistics. These differences are often associated with the properties of medium for optical signal propagation, with the system components characteristics and quantum nature of the optical radiation, for example, dispersion properties of optical units, defects and contamination of optical surfaces, the limited dynamic range of the photodetector, finite time of registration and many others $[12,13]$.

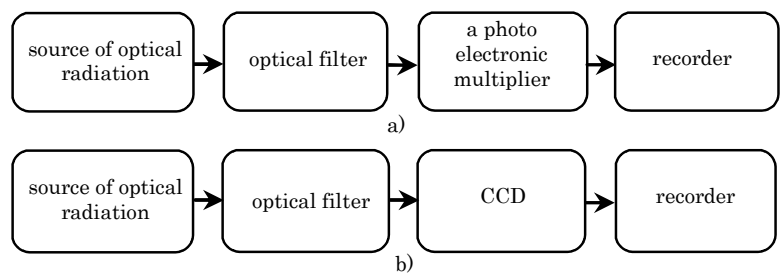

Fig. 1. Experimental installation.

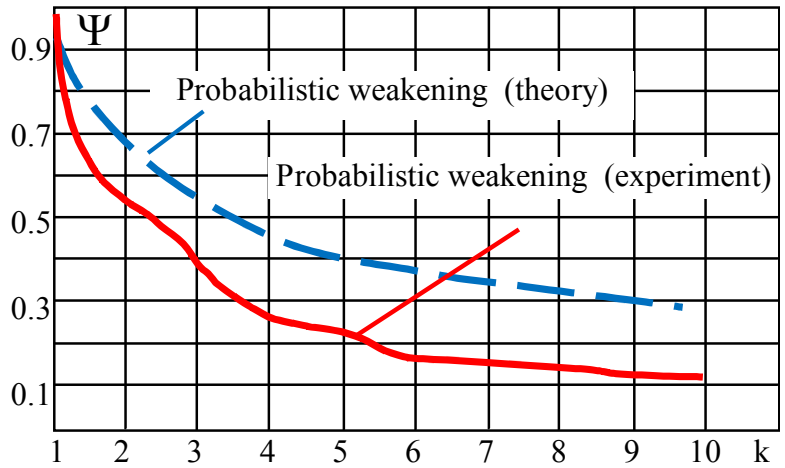

Fig. 2. Experimental and theoretical dependences of the normalized $\psi$ on the attenuation factor $k$. The Poisson model of optical radiation is adopted.

To clear up the reasons of deviations of the experimental results from the theoretical dependences, the statistical characteristics of signals were additionally studied and analyzed at different levels of weakening. The densities of output signals probabilities, asymptotical behavior of tails of the distribution densities and the limiting theorems for distribution of sums of independent random variables characterizing the output signal were defined.

The experiment, making it possible to study both temporal and spatial behavior of the output signal, was conducted with the use of the installation, shown in Fig. 1b. The optical signal, intensity of which was recorded by $\mathrm{CCD}$ in the range from 0 up to 255 brightness gradations, was supplied on the input of the system. The non-coherent sources of optical radiation, the neutral filters for matching the dynamic range were used. The video fragments with a frequency of record of 25 frames/s, resolution of $1000 \times 1000$ elements were examined frame-by-frame. It allowed to draw conclusions about sufficient spatial-temporal statistics and to accept the number of trials equal to $N \rightarrow \infty$.

Densities of output signals distribution without application of attenuators and with neutral filters in the system were obtained as a result of the experiments and presented in Fig. 3. 


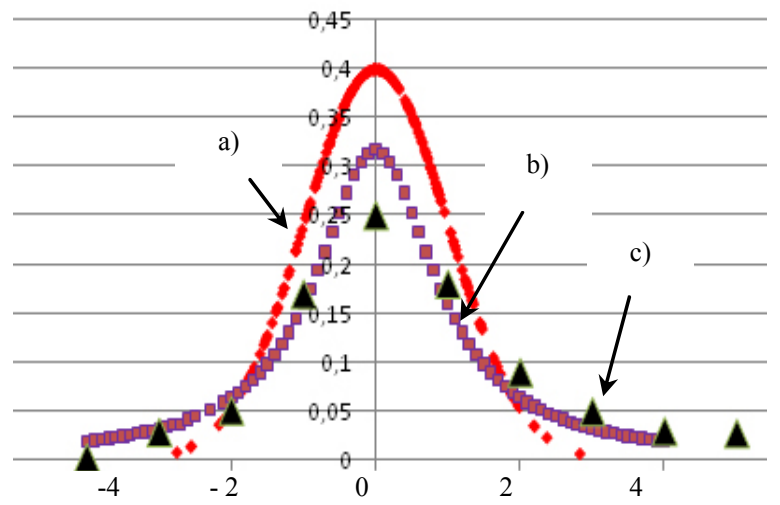

Fig. 3. a) Normal law of distribution; b) Cauchy distribution; c) Closeness of probability of weakened signals of the optoelectronic system with the neutral filter.

The limiting distributions characterizing the sum of identical distributied random variables are used when compiling algorithms for detection of random signals on the background of random noises. The process of formation of the optoelectronic system output signals is a sum of a large number of independent random variables, which, according to the central limiting theorem, gives the normal distribution law as the limiting one. When using the central limiting theorem, the elementary deviations resulting in the sum deviation are taken comparable in the order of their action on the sum dispersion.

Application of this mathematical apparatus makes it possible to obtain analytical expressions for the main indices of the system effectiveness and to create highly effective algorithms for signal processing in optoelectronic systems.

Approximation of the obtained densities of the optoelectronic system output signals distribution (without application of attenuators and with usage of neutral filters) with limiting distributions is presented in Figs 4 and 5.

The analysis of statistical behavior of the optoelectronic system output signals shows that asymptotical behavior of "tails" of the distribution density is not consistent with Gaussian statistics. This discrepancy can result in considerable errors when using the threshold methods of detection.

Studies of stochastic processes that do not submit to Gaussian statistics and classical central limiting theorem provoke great interest recently. The statistical feature of these processes consists in significantly higher probability of large fluctuations emergence. In these cases, it is necessary to use the generalized limiting theorems. Distribution of the sum of independent random variables is represented by the set of steady distributions [14].

As it follows from Figs 4 and 5, the optoelectronic system output signal is well approximated by the steady distribution laws.

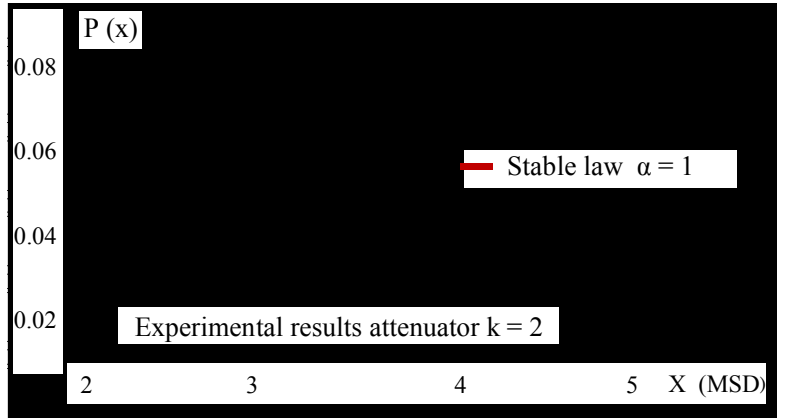

Fig. 4. Output signal is well approximated by the steady distribution law.

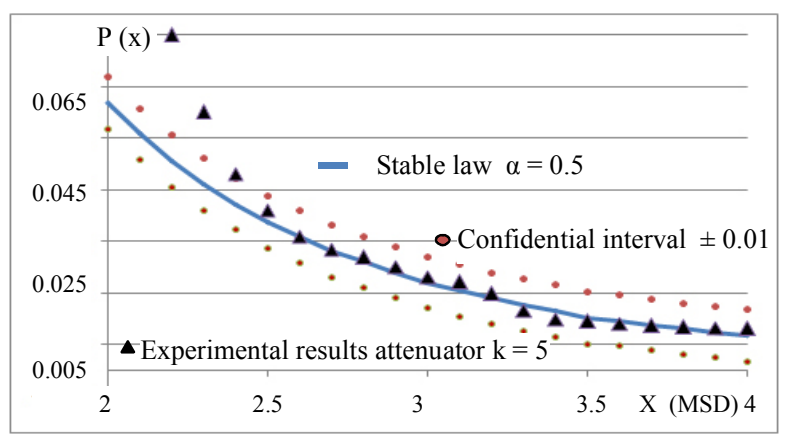

Fig. 5. Output signal is well approximated by the steady distribution law.

Table. Probability of appearance of events of the normalized and centered values.

\begin{tabular}{|c|c|c|c|c|c|}
\hline $\begin{array}{c}\mathrm{X}, \\
\text { MSD }\end{array}$ & $\begin{array}{c}\text { without } \\
\text { attenu- } \\
\text { ator }\end{array}$ & $\begin{array}{c}\text { Normal } \\
\text { distribu- } \\
\text { tion }\end{array}$ & $\begin{array}{c}\text { The } \\
\text { stable } \\
\text { laws } \\
\alpha=2\end{array}$ & $\begin{array}{c}\text { The } \\
\text { stable } \\
\text { laws } \\
\alpha=1\end{array}$ & $\begin{array}{c}\text { The } \\
\text { stable } \\
\text { laws } \\
\alpha=0.5\end{array}$ \\
\hline \multicolumn{6}{|c|}{ Probability of an event appearance } \\
\hline 2 & $4 \cdot 10^{-2}$ & $5 \cdot 10^{-2}$ & $1 \cdot 10^{-1}$ & $6 \cdot 10^{-2}$ & $5 \cdot 10^{-2}$ \\
\hline 3 & $3 \cdot 10^{-3}$ & $3 \cdot 10^{-3}$ & $3 \cdot 10^{-2}$ & $3 \cdot 10^{-2}$ & $1 \cdot 10^{-2}$ \\
\hline 4 & $5 \cdot 10^{-4}$ & $1 \cdot 10^{-4}$ & $5 \cdot 10^{-3}$ & $1 \cdot 10^{-2}$ & $6 \cdot 10^{-3}$ \\
\hline 5 & $1.6 \cdot 10^{-4}$ & $1.4 \cdot 10^{-6}$ & $5 \cdot 10^{-4}$ & $1 \cdot 10^{-2}$ & $3 \cdot 10^{-3}$ \\
\hline
\end{tabular}

\begin{tabular}{|c|c|c|c|c|c|}
\hline $\begin{array}{c}\mathrm{X}, \\
\text { MSD }\end{array}$ & $\begin{array}{c}\text { Attenu- } \\
\text { ator } \\
k=5\end{array}$ & $\begin{array}{c}\text { Normal } \\
\text { distribu- } \\
\text { tion }\end{array}$ & $\begin{array}{c}\text { The } \\
\text { stable } \\
\text { laws } \\
\alpha=2\end{array}$ & $\begin{array}{c}\text { The } \\
\text { stable } \\
\text { laws } \\
\alpha=1\end{array}$ & $\begin{array}{c}\text { The } \\
\text { stable } \\
\text { laws } \\
\alpha=0.5\end{array}$ \\
\hline \multicolumn{6}{|c|}{ Probability of an event appearance } \\
\hline 2 & $8 \cdot 10^{-2}$ & $5 \cdot 10^{-2}$ & $1 \cdot 10^{-1}$ & $6 \cdot 10^{-2}$ & $5 \cdot 10^{-2}$ \\
\hline 3 & $3.5 \cdot 10^{-2}$ & $3 \cdot 10^{-3}$ & $3 \cdot 10^{-2}$ & $3 \cdot 10^{-2}$ & $1 \cdot 10^{-2}$ \\
\hline 4 & $1 \cdot 10^{-2}$ & $1 \cdot 10^{-4}$ & $5 \cdot 10^{-3}$ & $1 \cdot 10^{-2}$ & $6 \cdot 10^{-3}$ \\
\hline 5 & $1 \cdot 10^{-2}$ & $1.4 \cdot 10^{-6}$ & $5 \cdot 10^{-4}$ & $1 \cdot 10^{-2}$ & $3 \cdot 10^{-3}$ \\
\hline
\end{tabular}

\begin{tabular}{|c|c|c|c|c|c|}
\hline $\begin{array}{c}\mathrm{X}, \\
\text { MSD }\end{array}$ & $\begin{array}{c}\text { Attenu- } \\
\text { ator } \\
k=10\end{array}$ & $\begin{array}{c}\text { Normal } \\
\text { distribu- } \\
\text { tion }\end{array}$ & $\begin{array}{c}\text { The } \\
\text { stable } \\
\text { laws } \\
\alpha=2\end{array}$ & $\begin{array}{c}\text { The } \\
\text { stable } \\
\text { laws } \\
\alpha=1\end{array}$ & $\begin{array}{c}\text { The } \\
\text { stable } \\
\text { laws } \\
\alpha=0.5\end{array}$ \\
\hline \multicolumn{5}{|c|}{ Probability of an event appearance } \\
\hline 2 & $8 \cdot 10^{-2}$ & $5 \cdot 10^{-2}$ & $1 \cdot 10^{-1}$ & $6 \cdot 10^{-2}$ & $5 \cdot 10^{-2}$ \\
\hline 3 & $1 \cdot 10^{-2}$ & $3 \cdot 10^{-3}$ & $3 \cdot 10^{-2}$ & $3 \cdot 10^{-2}$ & $1 \cdot 10^{-2}$ \\
\hline 4 & $8 \cdot 10^{-3}$ & $1 \cdot 10^{-4}$ & $5 \cdot 10^{-3}$ & $1 \cdot 10^{-2}$ & $6 \cdot 10^{-3}$ \\
\hline 5 & $5 \cdot 10^{-3}$ & $1.4 \cdot 10^{-6}$ & $5 \cdot 10^{-4}$ & $1 \cdot 10^{-2}$ & $3 \cdot 10^{-3}$ \\
\hline
\end{tabular}


Stochastic behavior of the output signals can be defined on the basis of the model of the input radiation photons interaction with an optical link of the optoelectronic system. Basically, the description and prediction of the optical link properties are based on definition of the deterministic parameters, i.e. on the coefficients of refraction, reflection, absorption. But accounting of random processes, taking place after the absorption of light quanta, photons bunching and antibunching, photon-phononic interaction and also chaotically located defects of a glass formed in the process of atmospheric effects and aging, location of color centers in optically dense materials makes it possible to interpret the obtained results more adequately.

The comparative analysis of probabilities of appearance of events of the normalized and centered values for the experimental data and limiting distributions is presented in Table.

\section{Discussion}

The Poisson and Gaussian models used for describing the output signals are in good agreement with the experimental data in the range up to $4 \mathrm{MSD}$ for the systems that do not use the attenuators. In the range from $5 \mathrm{MSD}$ and more, asymptotic behavior of output signal distribution law becomes closer to $\alpha$ - stable law with characterization index $\alpha=2$. Obviously, using the Poisson and Gaussian models with detection of objects will cause errors, if the detection threshold would be chosen in the range exceeding 5 MSD.

Asymptotic behavior of distribution functions of output signals in optical electronic systems with limited dynamic range is varying depending on neutral filter absorption factor. With growing the absorption factor, the output signal probability densities fall in the domain of attraction of the stable laws with characterization index $0<\alpha<2$.

Thus, when registering optical radiation with optoelectronic systems, the model of output signals built on the stable laws should be accepted. Using this model will allow to avoid conflict between experimental data and common mathematical models of output signals, and can serve as a theoretical basis for creation of high efficient algorithms of signal processing.

\section{References}

1. I.A. Cunninghom, R. Shaw, Signal-to-noise optimizatiom of medical imaging systems // J. Opt. Soc. Am. A, 19, p. 621-632 (1999).

2. A. Strelkov, Ye. Zhilin, A. Lytyuga, S. Lisovenko, Signal detection in techical vision systems // Telecom. and Radio Eng. 66, p. 283-293 (2007).

3. T. Strelkova, Influence of video stream compression on image microstructure in medical systems // Biomed. Eng. 47, p. 307-311 (2014).

4. D. Cokes, P. Lewis, Stochastic Analysis of Chains of Events. Mir Publ., Moscow, 1969.

5. A. Lytyuga, Mathematical model of signals in television systems with low-orbit space objects observation in daytime // Collected Works of Kharkiv University of Air Force, 4(22), p. 41-46 (2009).

6. F. Yang, Y.M. Lu, L. Sbaiz, M. Vetterli, Bits from photons. Oversampled image acquistion using binary Poisson statistics // IEEE Trans. Image Proc. 21, p. 1421-1436 (2012).

7. G. Bedard, Analysis of light fluctuations from photon counting statistics // Opt. Soc. Am. 57, p. 1201-1203 (1967).

8. A. Strelkov, Ye. Zhilin, T. Strelkova, A. Lytyuga, T. Butrim, Features of mathematical description of the optical radiation weakening processes // Radiotekhnika: All-Ukr. Sci. Interdep. Mag. 168, p. 97-102 (2012).

9. D. Smirnov, A. Troshin, New phenomena in quantum optics: Photon antibunching, sub-Poisson photon statistics, and squeezed states // Sov. Phys. Usp. 30, p. 851-874 (1987).

10. I. Bolshakov, Applied Theory of Random Fluxes. Nauka, Moscow, 1978.

11. V. Nikitin, V. Fomin, A. Borisenkov, A. Nikolaev, I.L. Borisenkov, Adaptive Noise Protection of Opticalelectronic Information Systems. Belgorod, 2008.

12. Jean M. Bennett, Lars Mattsson, Surface Roughness and Scattering. Optical Society of America, 1993.

13. T. Strelkova, Statistical properties of output signals in optical-television systems with limited dynamic range // Eastern-European Journal of Enterprise Technologies, 9(68), p. 38-44 (2014).

14. V.M. Zolotarev, Stable Laws and their Application. Mir Publ., Moscow, 1984. 